\title{
WEAK ASYMPTOTIC EQUIVALENCE RELATION AND INVERSE FUNCTIONS IN THE CLASS OR
}

\section{Dragan Đurčić and Aleksandar Torgašev*}

\begin{abstract}
If $f(x)$ is a continuous, strictly increasing and unbounded function defined on an interval $[a,+\infty)(a>0)$, in this paper we shall prove that $f^{-1}(x)(x \geq a)$ belongs to the Karamata class $O R$ of all $\mathcal{O}$-regularly varying functions, if and only if for every function $g(x)(x \geq a)$ which satisfies $f(x) \asymp g(x)$ as $x \rightarrow+\infty$, we have $f^{-1}(x) \asymp g^{-1}(x)$ as $x \rightarrow+\infty$. Here, $\asymp$ is the weak asymptotic equivalence relation. We shall also prove some variants of the previous theorem, in which, except the weak, we also deal with the strong asymptotic equivalence relation.
\end{abstract}

\section{Introduction and results}

A measurable function $f:[a,+\infty) \mapsto(0,+\infty)(a>0)$ is called $\mathcal{O}_{-}$ regularly varying in the Karamata sense [1], if it satisfies

$$
\varlimsup_{x \rightarrow+\infty} \frac{f(\lambda x)}{f(x)}=k_{f}(\lambda)<+\infty, \quad \lambda>0 .
$$

The class of all such functions is denoted $O R$, and as is well known, this class is one of the essential objects in the qualitative analyse of divergent asymptotic processes [1].

An $\mathcal{O}$ - regularly varying function $f:[a,+\infty) \mapsto(0,+\infty)(a>0)$, is called slowly varying in the Karamata sense [1], if it satisfies

$$
k_{f}(\lambda)=1, \quad \lambda>0 .
$$

The class of all such functions is denoted $S V$, and it is the most important object in the Karamata theory of regular variability [3].

AMS (MOS) Subject Classification 1991. Primary: 26A12.

Key words and phrases: Regular variation, Asymptotic equivalence, Inversion.

*Research supported by Science Fund of Serbia under Grant 1457. 
Two positive functions $f(x), g(x)(x \geq a)(a>0)$, are called weakly asimptoticaly equivalent, and denoted $f(x) \asymp g(x)$ as $x \rightarrow+\infty$, if there is some $\varepsilon>1$ such that

$$
\frac{1}{\varepsilon} \leq \frac{f(x)}{g(x)} \leq \varepsilon, \quad x \geq x_{0}(\varepsilon) .
$$

Next, they are called strongly asymptotically equivalent, and denoted $f(x) \sim g(x)$ as $x \rightarrow+\infty$, if (3) is satisfied for every $\varepsilon>1$.

Next, let $\mathcal{A}$ be the class of all positive functions, defined for $x \geq a$, for a fixed $a>0$, which are continuous, increasing and unbounded on the interval $[a,+\infty)$.

Assume that $f$ and $g$ are two functions from the class $\mathcal{A}$. We shall discuss some conditions under which we have that we have (4) implies (5), where (4) and (5) are the next relations:

$$
\begin{gathered}
f(x) \rho_{1} g(x), \quad x \rightarrow+\infty, \\
f^{-1}(x) \rho_{2} g^{-1}(x), \quad x \rightarrow+\infty,
\end{gathered}
$$

and $\rho_{1}$ and $\rho_{2}$ are some relations from the set $\{\asymp, \sim\}$.

We notice that the case $\rho_{1}=\rho_{2}=\sim$ is considered in the paper [2].

Theorem 1. (a) Suppose that $f$ and $g$ are two functions from the class $\mathcal{A}$, next at least one of the functions $f^{-1}, g^{-1}$ belongs to the class $O R$, and relation (4) is satisfied for $\rho_{1}=\asymp$. Then the relation (5) is also true with $\rho_{2}=\asymp$.

(b) If $f \in \mathcal{A}$, and every function $g \in \mathcal{A}$ which satisfies (4) with $\rho_{1}=\asymp$ also satisfies (5) with $\rho_{2}=\asymp$, then $f^{-1} \in O R$, and $g^{-1} \in O R$.

Theorem 2. (a) Suppose that $f, g \in \mathcal{A}$, next at least on of the functions $f^{-1}, g^{-1}$ belongs to the class $S V$, and relation (4) is true for $\rho_{1}=\asymp$. Then the relation (5) is also true with $\rho_{2}=\sim$.

(b) If $f \in \mathcal{A}$ and for every function $g \in \mathcal{A}$ which satisfies (4) for $\rho_{1}=\asymp$, (5) is also true with $\rho_{2}=\sim$, then $f^{-1} \in S V$, and $g^{-1} \in S V$.

Theorem 3. (a) Suppose that $f, g \in \mathcal{A}$, next at least one of the functions $f^{-1}, g^{-1} \in O R$, and relation (4) is true for $\rho_{1}=\sim$. Then the relation (5) is also true for $\rho_{2}=\asymp$.

(b) If $f \in \mathcal{A}$, and for every function $g \in \mathcal{A}$ which satisfies (4) with $\rho_{1}=\sim$, (5) is also true with $\rho_{2}=\asymp$, then $f^{-1} \in O R$ and also $g^{-1} \in O R$.

We notice that previous theorems are in fact some characterizations of the Karamata classes $O R \cap \mathcal{A}$ and $S V \cap \mathcal{A}$. 


\section{Proofs of theorems}

Proof of Theorem 1. (a) Without loos of generality, we can assume that the function $g^{-1} \in O R$. By relation $f(x) \asymp g(x)(x \rightarrow+\infty)$, we have that $\underline{\lim }_{x \rightarrow+\infty} \frac{f(x)}{g(x)}=m>0$. Therefore, there is a $\lambda_{1}>\frac{1}{m}$ such that $f(x) \geq$ $\frac{1}{\lambda_{1}} g(x)$ for $x \geq x_{0}\left(\lambda_{1}\right)$. Thus, for all enough large $x$ we have

Hence we get

$$
\frac{f^{-1}(x)}{g^{-1}(x)} \leq \frac{g^{-1}\left(\lambda_{1} x\right)}{g^{-1}(x)} \text {. }
$$

$$
\varlimsup_{x \rightarrow+\infty} \frac{f^{-1}(x)}{g^{-1}(x)} \leq k_{g^{-1}}\left(\lambda_{1}\right)<+\infty .
$$

Besides, we have that $\varlimsup_{x \rightarrow+\infty} \frac{f(x)}{g(x)}=M<+\infty$. Therefore, there is a positive number $\lambda_{2}<\frac{1}{M}$ such that $f(x) \leq \frac{1}{\lambda_{2}} g(x)$, for $x \geq x_{0}\left(\lambda_{2}\right)$. This means that for all enough large $x$ we have

Hence, we find that

$$
\frac{f^{-1}(x)}{g^{-1}(x)} \geq \frac{g^{-1}\left(\lambda_{2} x\right)}{g^{-1}(x)} .
$$

$$
\underline{\lim }_{x \rightarrow+\infty} \frac{f^{-1}(x)}{g^{-1}(x)} \geq \frac{1}{k_{g^{-1}}\left(1 / \lambda_{2}\right)}>0 .
$$

Therefore, we have that $f^{-1}(x) \asymp g^{-1}(x)$ as $x \rightarrow+\infty$.

(b) Suppose that $f \in \mathcal{A}$. Let $\lambda>0$ and $g(x)=\lambda \cdot f(x)(x \geq a, a>0)$. Then we have that $g \in \mathcal{A}$ and $f(x) \asymp g(x), x \rightarrow+\infty$. Therefore, $f^{-1}(x) \asymp$ $g^{-1}(x), x \rightarrow+\infty$, so that

$$
\begin{aligned}
+\infty>A(\lambda) & \geq \varlimsup_{x \rightarrow+\infty} \frac{f^{-1}(x)}{g^{-1}(x)}= \\
& =\varlimsup_{t \rightarrow+\infty} \frac{f^{-1}(g(t))}{g^{-1}(g(t))}= \\
& =\varlimsup_{t \rightarrow+\infty} \frac{f^{-1}(\lambda f(t))}{t}= \\
& =\varlimsup_{t \rightarrow+\infty} \frac{f^{-1}(\lambda f(t))}{f^{-1}(f(t))}= \\
& =\varlimsup_{p \rightarrow+\infty} \frac{f^{-1}(\lambda p)}{f^{-1}(p)}= \\
& =k_{f^{-1}}(\lambda), \quad \lambda>0 .
\end{aligned}
$$


Hence, the function $f^{-1} \in O R$. Besides, for every function $g \in \mathcal{A}$ for which $f(x) \asymp g(x), x \rightarrow+\infty$, we have that $g^{-1}(x)=h(x) \cdot f^{-1}(x)$ for $0<\frac{1}{A(g)} \leq h(x) \leq A(g)<+\infty$ if $x \geq x_{0}(g)$. Therefore,

$$
k_{g^{-1}}(\lambda) \leq k_{f^{-1}}(\lambda) \cdot A^{2}(g)<+\infty, \quad \lambda>0 .
$$

Hence, $g \in O R$.

Theorem 2 can be proved analogously as the Theorem 1, and the Theorem 3 (a) is a direct consequence of the Theorem 1 (a). So, we shall only prove Theorem $3(\mathrm{~b})$.

Proof of Theorem 3. (b) Suppose that $f \in \mathcal{A}$. Then $k_{f^{-1}}(\lambda) \leq 1$ for $0<\lambda \leq 1$. Next notice that if $g \in \mathcal{A}$ and $f(x) \sim g(x), x \rightarrow+\infty$, then $f^{-1}(x) \asymp g^{-1}(x), x \rightarrow+\infty$, so that

$$
\begin{aligned}
+\infty>A(g) & \geq \varlimsup_{x \rightarrow+\infty} \frac{f^{-1}(x)}{g^{-1}(x)}= \\
& =\varlimsup_{t \rightarrow+\infty} \frac{f^{-1}(g(t))}{g^{-1}(g(t))}= \\
& =\varlimsup_{t \rightarrow+\infty} \frac{f^{-1}(g(t))}{f^{-1}(f(t))} .
\end{aligned}
$$

Next, let $\alpha(t)(t \geq a ; a>0)$ be an arbitrary positive continuous function such that $\alpha(t) \geq 1$ and $\alpha(t) \rightarrow 1+$ for $t \rightarrow+\infty$. We shall discuss the function $\beta(t)=\alpha(f(t))$ for $t \geq a$. If the function $h(t)=\beta(t) f(t), t \geq a$, is increasing, then $h \in \mathcal{A}$ and we have $f(t) \sim h(t)$ as $t \rightarrow+\infty$. Hence we get

$$
\begin{aligned}
+\infty>A(h) & \geq \varlimsup_{t \rightarrow+\infty} \frac{f^{-1}(\beta(t) f(t))}{f^{-1}(f(t))}= \\
& =\varlimsup_{t \rightarrow+\infty} \frac{f^{-1}(\alpha(f(t)) f(t))}{t}= \\
& =\varlimsup_{p \rightarrow+\infty} \frac{f^{-1}(\alpha(p) \cdot p)}{f^{-1}(p)} .
\end{aligned}
$$

If $h(t), t \geq a$, is not increasing, then we can consider the function $r(t)=\max _{a \leq x \leq t} h(x), t \geq a$. This function is continuous, nondecreasing and satisfies $r(t) \rightarrow+\infty, t \rightarrow+\infty$, and $r(t) \geq \beta(t) \cdot f(t), t \geq a$. Let $\varepsilon>0$. Then there is a $t_{0} \geq a$ such that

$$
1 \leq h(t) / f(t)<1+\varepsilon, \quad t \geq t_{0},
$$

and next there is a $t_{1}>t_{0}$ such that

$$
h(t) \geq \max _{a \leq u \leq t_{0}} h(u),
$$


for all $t \geq t_{1}$. Then for every $t \geq t_{1}$ and a function $v(t) \in\left[t_{0}, t_{1}\right]$ we have that

$$
\begin{aligned}
1 & \leq \frac{r(t)}{f(t)}=\frac{1}{f(t)} \max _{a \leq u \leq t} h(u)= \\
& =\frac{1}{f(t)} \max _{t_{0} \leq u \leq t} h(u)=\frac{h(v(t))}{f(t)} \leq \\
& \leq \frac{h(v(t))}{f(v(t))}<1+\varepsilon .
\end{aligned}
$$

Hence we get $r(t) \sim f(t), t \rightarrow+\infty$. Define next the function $r_{1}(t)$, $t \geq a$, with $r_{1}(t)=r(t)+u(t)$, where $u(t), t \geq a$ is an increasing, continuous function such that $u(t) \rightarrow 1-, t \rightarrow+\infty$. Then $r_{1} \in \mathcal{A}$ and we have that $r_{1}(t) \sim r(t) \sim f(t), t \rightarrow+\infty$. Therefore we find that

$$
\varlimsup_{t \rightarrow+\infty} \frac{f^{-1}(\beta(t) f(t))}{f^{-1}(f(t))} \leq \varlimsup_{t \rightarrow+\infty} \frac{f^{-1}\left(r_{1}(t)\right)}{f^{-1}(f(t))} \leq A\left(r_{1}\right)<+\infty .
$$

Hence,

$$
\begin{array}{r}
\varlimsup_{t \rightarrow+\infty} \frac{f^{-1}(\beta(t) f(t))}{f^{-1}(f(t))}=\varlimsup_{t \rightarrow+\infty} \frac{f^{-1}(\alpha(f(t)) f(t))}{f^{-1}(f(t))}= \\
=\varlimsup_{p \rightarrow+\infty} \frac{f^{-1}(\alpha(p) p)}{f^{-1}(p)} \leq A\left(r_{1}\right)<+\infty .
\end{array}
$$

Now we shall prove that $\varlimsup_{\substack{\lambda \rightarrow 1+\\ x \rightarrow+\infty}} \frac{f^{-1}(\lambda x)}{f^{-1}(x)}=A<+\infty$, where $A$ is a finite real number.

On the contrary, suppose that there are some sequences $\left(\lambda_{n}\right),\left(x_{n}\right)$ such that $\lambda_{n} \rightarrow 1+$ and $x_{n} \rightarrow+\infty$ as $n \rightarrow \infty$, such that

$$
\frac{f^{-1}\left(\lambda_{n} x_{n}\right)}{f^{-1}\left(x_{n}\right)} \rightarrow+\infty, \quad n \rightarrow \infty .
$$

Without loss of generality, we can assume that $x_{n} \geq a(n \in N)$, next that $\left(x_{n}\right)$ is an increasing sequence, and that $\lambda_{n} \geq 1$ for every $n \in N$. Define a function $\alpha(x), x \geq a$, with $\alpha\left(x_{n}\right)=\lambda_{n}(n \in N)$, and $\alpha(x)=\lambda_{1}$ for $x \in\left[a, x_{1}\right)$, and on the interval $\left(x_{k}, x_{k+1}\right)(k \geq 1)$ take the usual linear and continuous extension. The so obtained function $\alpha:[a,+\infty) \mapsto[1,+\infty)$ is continuous, and we have $\alpha(x) \rightarrow 1+$ as $x \rightarrow+\infty$. Consequently, we get

$$
\varlimsup_{n \rightarrow+\infty} \frac{f^{-1}\left(\alpha\left(x_{n}\right) x_{n}\right)}{f^{-1}\left(x_{n}\right)}=\varlimsup_{n \rightarrow+\infty} \frac{f^{-1}\left(\lambda_{n} x_{n}\right)}{f^{-1}\left(x_{n}\right)}=+\infty,
$$

what is a contradiction to $(6)$. 
Hence, for every $\varepsilon>0$ there is an $x_{0} \geq a$ and a $\delta>0$, so that for all $x \geq x_{0}$ and all $\lambda \in[1,1+\delta]$, we have

$$
1 \leq \frac{f^{-1}(\lambda x)}{f^{-1}(x)} \leq A+\varepsilon
$$

Thus, if $\lambda \in(0,1+\delta]$ we have that $k_{f^{-1}}(\lambda) \leq A+\varepsilon<+\infty$. Since $f^{-1}$ is increasing, we have that $k_{f^{-1}}(\lambda)<+\infty$ for all $\lambda>0$ (see e.g. [3]), so we find that $f^{-1} \in O R$.

The remaining part of the proof coincides with the corresponding part of the proof of Theorem 1 (b).

Corollary. Assume that both $f, g \in \mathcal{A}$.

(a) If at least one of the functions $f^{-1}, g^{-1} \in O R$, and (4) holds for $\rho_{1}=\asymp$ or $\rho_{1}=\sim$, then both functions $f^{-1}, g^{-1} \in O R$.

(b) If at least one of the functions $f^{-1}, g^{-1} \in S V$, and (4) is true for $\rho_{1}=\asymp$ or $\rho_{1}=\sim$, then both functions $f^{-1}, g^{-1} \in S V$.

\section{References}

[1] N. H. Bingham, C. M. Goldie, J. L. Teugels: Regular Variation, Cambridge Univ. Press, Cambridge, 1987.

[2] D. Đurčić, A. Torgašev: Strong Asymptotic Eqivalence and Inversion of Functions in the class $K_{c}$, Journal Math. Anal. Appl. 255 (2001), 283-290.

[3] E. Seneta: Regularly varying functions, Lecture Notes in Math. No. 508, SpringerVerlag, Berline, 1976.

First author:

Technical Faculty, Svetog Save 65, 32000 Čačak, Serbia \& Montenegro.

Second author: Mathematical Faculty, Studentski trg 16a, 11000 Belgrade,

Serbia \& Montenegro 